\title{
Localized Liquid Secretion from a Photopatterned Liquid-Crystal Polymer Skin
}

Yuanyuan Zhan, Wanshu Zhang, Ayberk Özden, Simon Houben, Margarita Schuster, Hubert Gojzewski, Guofu Zhou,* Dirk J. Broer, and Danqing Liu*

Cite This: ACS Appl. Polym. Mater. 2020, 2, 4071-4077

Read Online

ABSTRACT: Liquid-releasing artificial skins are made from films made of a smectic liquid-crystal polymer network (LCN) photopolymerized in the presence of a photoactive azobenzene chromophore and a liquid-crystal porogen. The nonreactive porogen phase separates during the polymerization process, and the polymer forms a spongy polymer network filled with liquid. The liquid is excreted from the film when exposed to UV light upon conversion of trans-azobenzene to its cis isomer. Here, localized liquid secretion at preset positions at the polymer film is described. The design

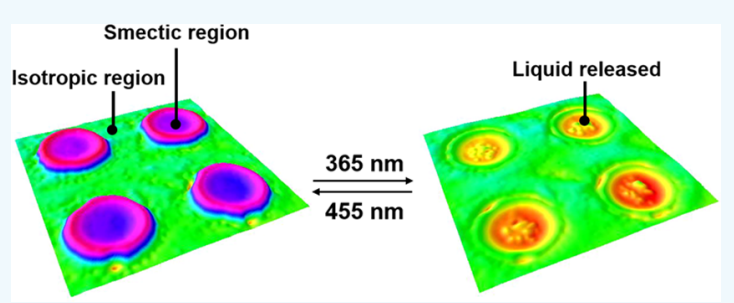
principle is based on creating a hybrid molecular architecture with both smectic and nonordered isotropic alignments in a continuous LCN coating. This coating is fabricated by a maskwise photopolymerization of the monomer mixture in the smectic phase, followed by a flood exposure at an elevated temperature above the isotropic state of the unpolymerized region. The smectic regions that polymerized during the maskwise exposure are not affected by the heating needed for the second polymerization step of the isotropic area. Upon activation under light illumination, the embedded liquid is exclusively released from the area with the smectic alignment. This approach reveals films of which the excretion process is reversible. The secreted liquid is reabsorbed spontaneously when azobenzene takes its initial trans-form. This process occurs thermally in time or can be accelerated by light irradiation with visible light.

KEYWORDS: liquid-crystal polymer network, liquid secretion, polymerization-induced diffusion, photoresponsive coating, localized release

\section{INTRODUCTION}

Excretion of substances from their surface is a vital biological process that protects organisms by forming defensive layers toward their environment, controlling temperature, transporting substances, and carrying out chemical reactions. ${ }^{1-5}$ For example, fish secrete slimy mucus from their skin to protect against parasites from attaching to the scales and to reduce friction with water to make them swim faster. ${ }^{3,6}$ In humans, skin excretes skin oil to defend the body against fungus and bacteria, ${ }^{7}$ and we sweat to regulate the body temperature. ${ }^{4}$ Many synthetic routes have been developed to mimic this natural phenomenon. One of the successful attempts to develop a self-regulated and interactive surface was reported by Aizenberg et al. in $2015 .^{8}$ Inspired by the selfstoring phenomenon from nature such as the liquid coating on our eyes, they demonstrated a coating that replenishes its surface with a stored lubricant. Driven by surface energy, a silicon oil-based lubricating liquid is released from a poly(dimethylsiloxane) (PDMS)-based porous polymer coating in which it is dissolved. Therefore, the coating surface continually adjusts its characteristics. The rejected lubricant covers the coating surface and forms an interfacial fouling-release layer, which makes the surfaces extremely slippery and repels unwanted surface attachments such as bacteria, algae, dust, and ice. ${ }^{9}$ Whether the spontaneous and continuous release is an advantage or a disadvantage depends on its application. ${ }^{10}$ Especially, where a regulated release is demanded, e.g., to control the lifetime of the effect or where the lubrication is needed only temporarily, new release strategies are required.

Recently, our group developed a "photosponge" based on liquid-crystal polymer networks (LCNs). ${ }^{11}$ Well-ordered pores loaded with liquid are formed in a liquid-crystal polymer coating. The porous structures can collapse and re-form, subsequently releasing and, reversibly, taking up fluid under the control of light. The released liquid changes and controls the tribological behavior of the coating surface. It was demonstrated that when the coating is brought into contact with an opposing surface the light-controlled release of fluid may induce capillary bridging, thus providing adhesion between the two coatings. The surface force tightly connects two coatings forming a temporary grip or brake, claimed to be of interest for soft robotic applications. Although this method successfully demonstrates administration and, reversibly, the

Received: June 24, 2020

Accepted: August 13, 2020

Published: August 13, 2020 

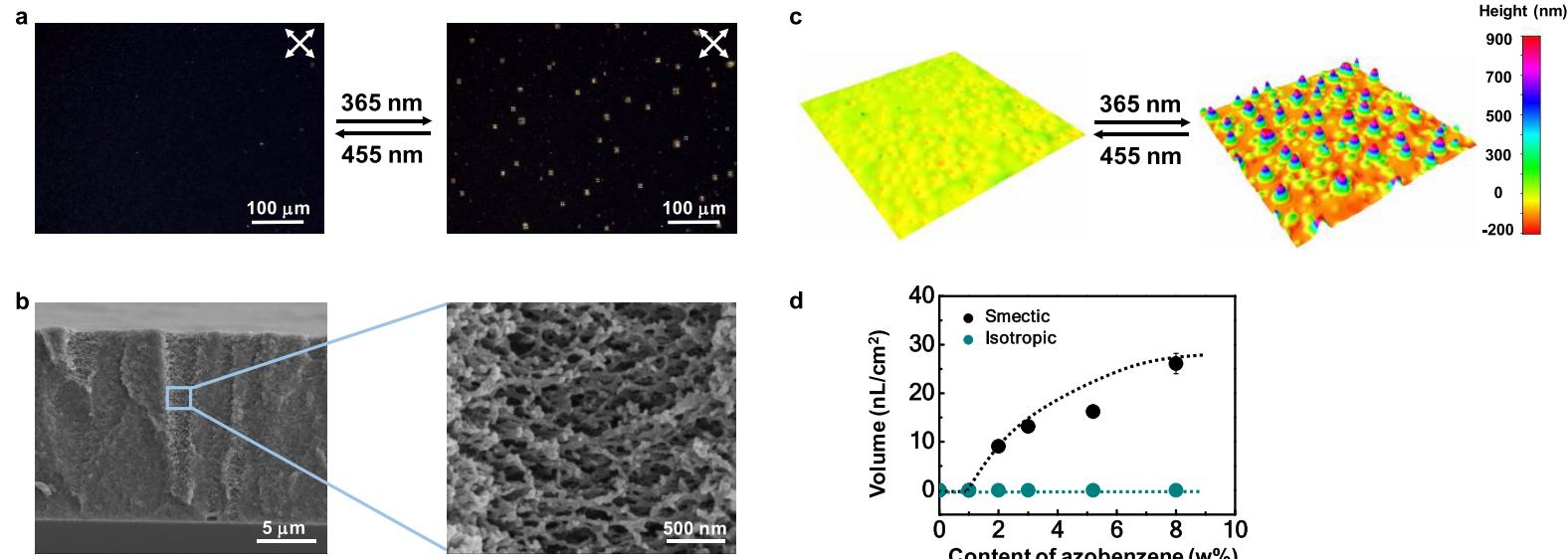

d

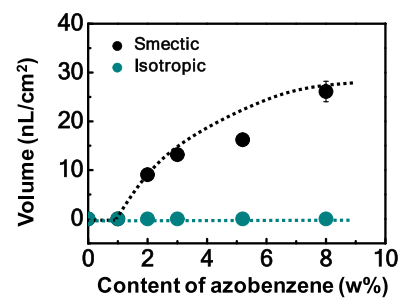

Figure 1. Light-responsive liquid-secreting LCN coating. (a) POM images showing the released birefringent liquid under UV illumination. (b) SEM pictures showing the initial porous structure of the coating after removal of 3 by solvent extraction. (c) DHM images showing the coating that repels liquid upon light illumination. (d) Influence of azobenzene (molecule 4) concentration and the molecular alignment on secretion.

uptake of the liquid, the released liquid is spread only uniformly over the entire coating surface, while the confined secretion at the preferred location in the micrometer range remains a challenge.

In this work, therefore, we advance the "photosponge" approach with the aim to spatially regulate the excretion at the surface. To this end, we design and fabricate a coating with hybrid molecular configurations, in which areas with a smectic alignment are next to regions with a nonordered isotropic order. When activated by light, liquid is exclusively ejected from the regions with the smectic alignment.

\section{RESULTS AND DISCUSSION}

In the first experiment, we produced a uniform microporous LCN coating on glass by photopolymerizing a blend of liquidcrystal monomers in the presence of a nonreactive liquid crystal in its smectic phase. An optimized composition with respect to the quantity of secreted liquid and related velocity is shown in the Materials and Methods section. We have chosen liquid-crystal diacrylate $\mathbf{1}$ and monoacrylate $\mathbf{2}$ to form the polymer network. The nonreactive liquid-crystal 3 stabilizes the smectic phase and acts as the phase-separating porogen. The azobenzene-containing monomer $\mathbf{4}$ or $\mathbf{5}$ not only provides light responsiveness but also acts as a cross-linker. LCNs with an integrated azobenzene cross-linker are known for their photomechanical deformation. ${ }^{12}$ Both stand-alone films and coatings confined at their substrate demonstrated lighttriggered morphing in shape and surface topography. ${ }^{13-25}$ Prior to its polymerization, the smectic mixture is aligned with its director perpendicular to the substrate, further defined as homeotropic orientation, by means of a polyimide alignment film at a glass substrate. This order is preserved upon photopolymerization at $35{ }^{\circ} \mathrm{C}$ by exposure to light of wavelength $>400 \mathrm{~nm}$ activating the added photoinitiator 6 . This relatively longer wavelength is chosen to avoid premature isomerization of trans-azobenzene during the polymerization process.

To study the secretion process of the "sponge", we fabricated a coating with a thickness of $20 \mu \mathrm{m}$. We characterized this coating by polarized optical microscopy (POM), as shown in Figure 1a. The image is black between two crossed polarizers and remains black upon rotating the sample, confirming that the homeotropic alignment is obtained. We performed scanning electron microscopy (SEM) measurements after the removal of porogens to analyze the morphology of the formed smectic film. We assume that after the extraction of 3 , the pores maintain their initial morphologies as a minor thickness reduction takes place (Supporting Information, Figure S1). From the SEM photos as presented in Figure $1 \mathrm{~b}$, we estimated the pore size to be approximately $100-200 \mathrm{~nm}$ in diameter.

Upon illuminating the coating with UV light, the initially "locked" liquid is rejected at the coating surface, visualized by POM (Figure 1a, Movie S1) and digital holographic microscopy (DHM) (Figure 1c, Movie S2), showing that the released liquid forms droplets uniformly distributed over the entire coating surface. The triggered liquid rejection utilizes the liquid-crystal properties of the network by its ability to change the molecular alignment and order (Figure 1d). Under UV irradiation, the copolymerized azobenzene (molecule 4) isomerizes from the trans- to cis-state and subsequently changes the order of the smectic LCN. This order reduction leads to a strong contraction along the coating thickness. Since the film strongly adheres to the glass substrate, lateral deformation is largely restricted. ${ }^{26}$ Therefore, the pore dimensions are reduced when squeezing out the initially locked liquids at the polymer skin surface. Assuming the pores collapse without leaving unfilled micro/nanosized voids, we can correlate the volume of the rejected liquid with the thickness reduction of the coating.

The amount of liquid that is secreted from the film depends on the concentration of the copolymerized azobenzene. Figure $1 \mathrm{~d}$ shows an increase in the amount of secreted liquid with increasing azobenzene content, which can be explained in terms of a higher density of contracting units per unit volume.

Next, in a reference experiment, we estimate the contribution of the molecular order to the secretion. We prepared a coating with the same composition, which was polymerized in the isotropic state at an elevated temperature of $75{ }^{\circ} \mathrm{C}$. The sample was examined under POM (Supporting Information, Figure S2); although the polymer network is isotropically aligned, the phase-separated porogen (molecule 3 ) in the pores is in the smectic phase. When triggered by UV light, the amount of liquid that is excreted at the coating surface from the isotropic $\mathrm{LCN}$ is far less than that released from the LCN with the smectic order (Figure 2a). Even at high 

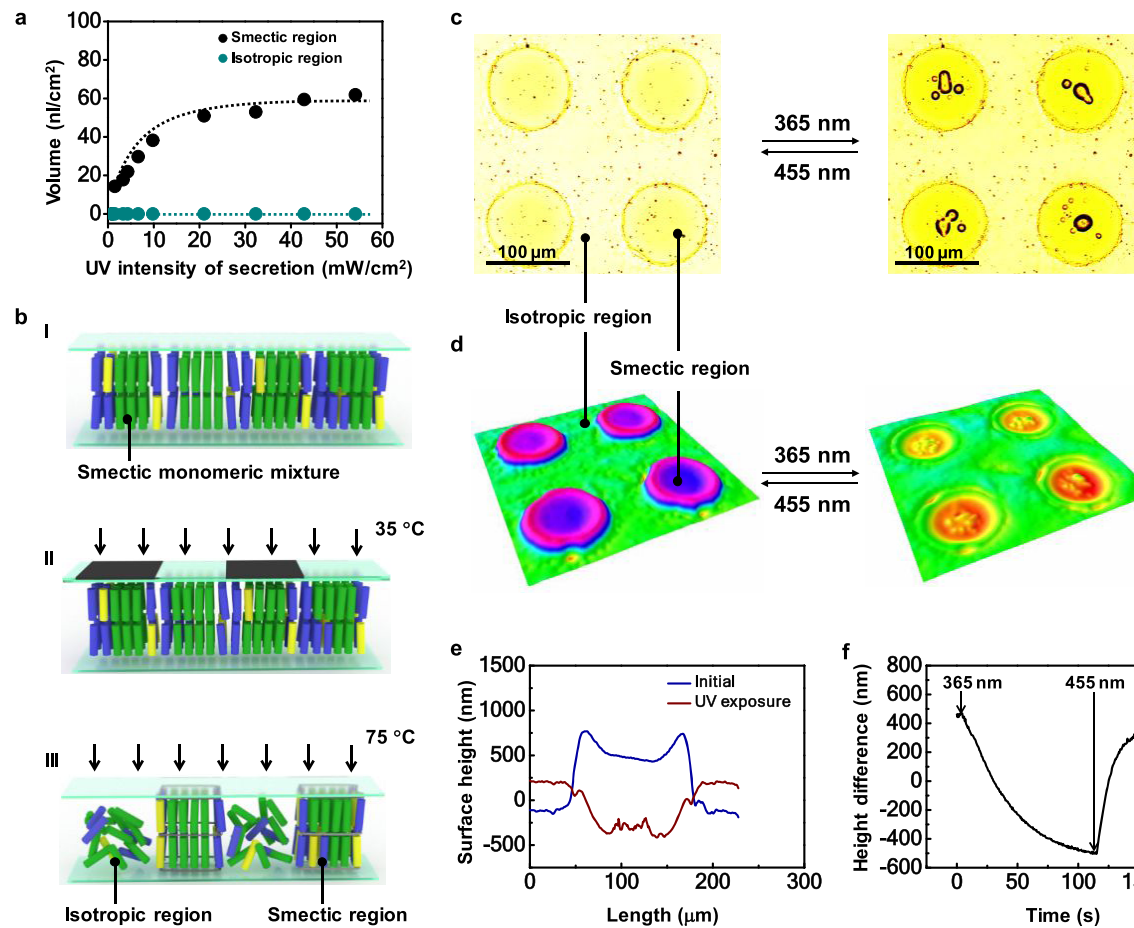

Figure 2. Localized secretion at the surface of a coating consisting of hybrid molecular configurations. (a) Comparison of the secreted liquid in smectic and isotropic regions under various UV intensities. (b) Schematic representation showing the procedure to fabricate the coating consisting of areas of both smectic and isotropic regions. (I) Mixture given in Scheme 1 is filled in a cell. (II) Localized exposure through a photomask is carried out at the smectic state of the monomer mixture. (III) Unpolymerized monomers are heated to the isotropic phase and polymerized during the flood exposure at $75{ }^{\circ} \mathrm{C}$. (c) Optical microscopic images showing the released liquid droplet formed in the area with the smectic molecular order. (d) 3D images measured by DHM showing the decrease of surface protrusion when the liquid is excreted. (e) Corresponding $2 \mathrm{D}$ profiles of (d). (f) Secretion kinetics measured as surface height change over time during liquid secretion. The applied UV intensity is $0.9 \mathrm{~mW} / \mathrm{cm}^{2}$.
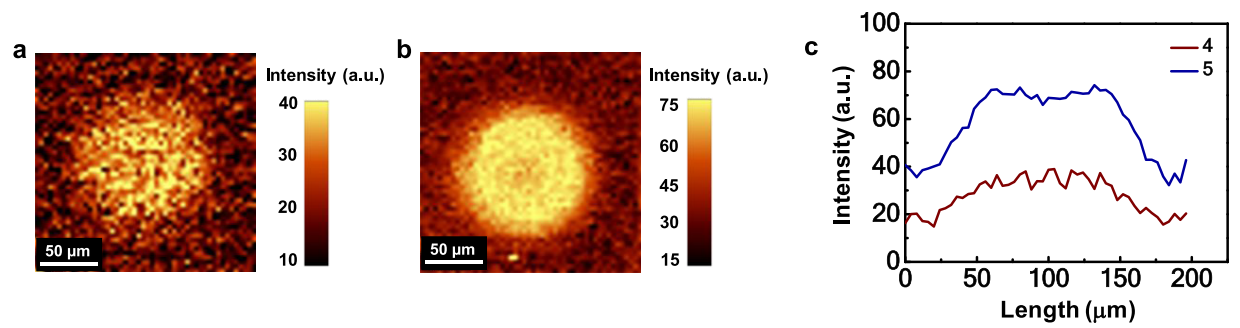

Figure 3. Confocal Raman measurements showing the distribution of azobenzene 4 (a) and 5 (b) after the first maskwise exposure and (c) corresponding $2 \mathrm{D}$ intensity profiles of $(\mathrm{a}, \mathrm{b})$.

azobenzene concentrations (Figure 1d), the secretion from isotropic LCNs is close to zero, indicating that the contracting force of the homeotropic-oriented smectic LCN is responsible for secretion.

With this acquired knowledge, we now create regions with a nonordered isotropic alignment in the smectic LCN film. The coating is fabricated by a two-step exposure process (Figure $2 b)$. In the first maskwise photopolymerization step, the monomer mixture in its smectic phase at $35{ }^{\circ} \mathrm{C}$ is selectively polymerized under the illuminated area (Figure $2 \mathrm{~b}$,II). The optimized exposure condition is $0.1 \mathrm{~mW} / \mathrm{cm}^{2}$ for $600 \mathrm{~s}$. During the second flood exposure, the coating is heated above the nematic-isotropic transition temperature $\left(T_{\mathrm{N}-\mathrm{I}}\right)$ at $75{ }^{\circ} \mathrm{C}$ of the unpolymerized materials (Figure 2a,III). The heating step does not affect the polymerized smectic phase but causes minor thermal phase separation of the nonreactive liquid molecule 3 from the polymer network at the coating surface, as observed from POM (Figure 2c). The film obtained consists of areas with both smectic and isotropic molecular alignments.

Prior to secretion, the smectic areas are protruded in comparison with the isotropic area due to the polymerizationinduced diffusion process, which is described later. When triggered by UV exposure, liquid molecule 3 is exclusively secreted from the area with the smectic molecular configuration as analyzed by an optical microscope (Figure 2c, Movie S3). From the DHM measurement, we observe that the secreting area decreases in height, indicating the stored liquid diminishes (Figure 2d,e, Movie S4). However, in the inactive area with the isotropic phase, the thickness slightly increases, most likely due to the sidewise diffusion from the smectic regions. The liquid secretion takes $100 \mathrm{~s}$ to reach equilibrium (Figure 2f), which is much faster compared with $4 \mathrm{~h}$ reported by others for nonactivated coatings. ${ }^{8}$ We further varied the UV intensity to control secretion, as seen in Figure 2a and Figure S3 in the Supporting Information. With increasing UV 

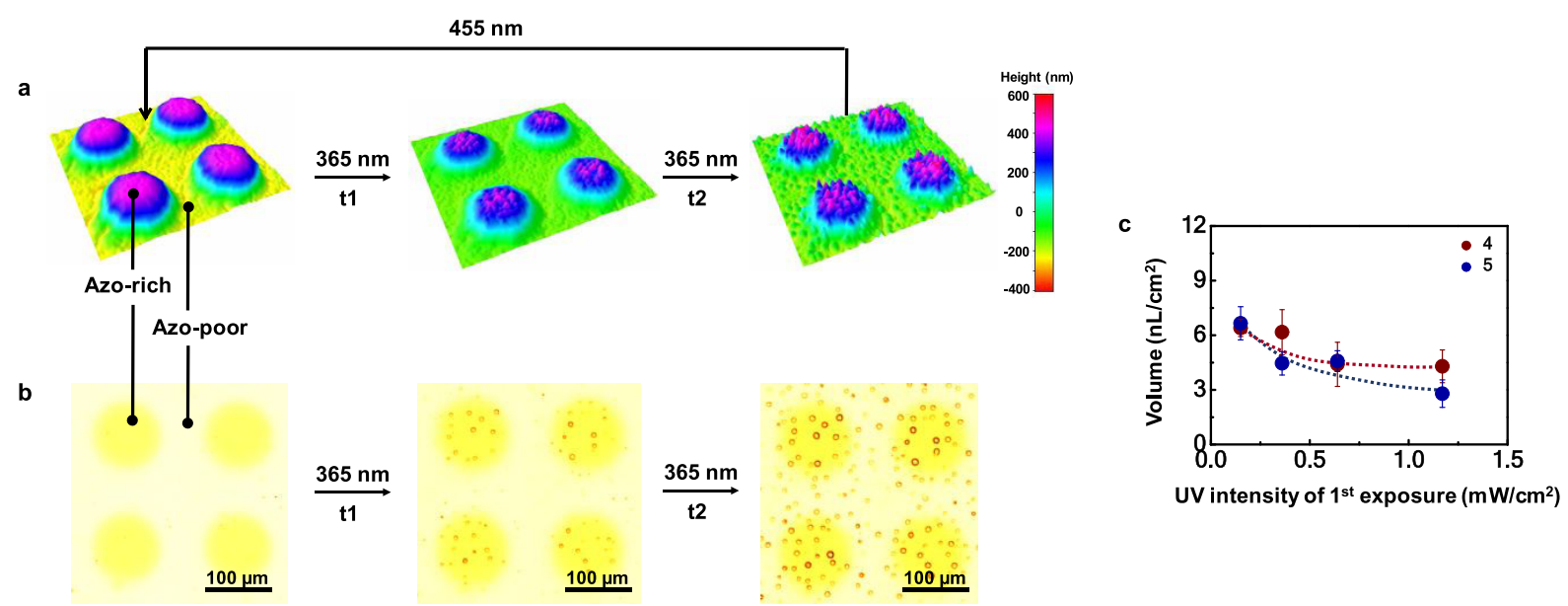

Figure 4. Liquid excretion in a patterned azobenzene coating. (a) 3D images measured by DHM showing the changes in the surface profile upon secretion over time. $t 1<t 2$, (b) optical microscopic pictures visualizing the formation of liquid droplets upon secretion, and (c) influence of the light intensity during the first mask-exposure step on the amount of secreted liquid. The UV intensity applied to trigger the secretion is $0.9 \mathrm{~mW} /$ $\mathrm{cm}^{2}$.

intensity, the amount of released liquid and the secretion speed increase. We kept the exposure time constant at $66 \mathrm{~s}$, implying that the intensity can also be read as dose.

Noticeably, DHM measurements reveal some elevation in the smectic area accompanied by deeper ridges at the edges (Figure 2d). This is a typical phenomenon inherent to the first maskwise photopolymerization and originates from the polymerization-induced diffusion process. ${ }^{27}$ This process forms the base of photoembossed surface topographies ${ }^{28-31}$ and is used to create a 3D morphing film in hydrogels. ${ }^{32,33}$ In a simplified model, polymerization-induced diffusion is initiated by local differences in light intensity during the photopolymerization process in a monomer mixture containing monomers with different polymerization and/or diffusion rates. $^{28,34}$ It is therefore expected that the smectic area is enriched by a higher concentration of the responsive azobenzene molecules, possibly at the expense of some of the nonreactive molecule 3 .

To study the polymerization-induced diffusion of the reactive components during the first maskwise exposure step, we performed confocal Raman experiments (Figure 3). In the Raman spectra of the blends, we focused on the $\mathrm{N}=\mathrm{N}$ stretch vibration at $1410 \mathrm{~cm}^{-1}$, representing the azobenzene molecule 4 or 5 , and on the nitrile stretch vibration at $2226 \mathrm{~cm}^{-1}$, representing component 3 (Supporting Information, Figure S4). The spatially resolved ratio between the Raman intensities is a measure for the relative concentration of both components. As an example of a measurement, Figure $3 a, c$ shows an intensity plot demonstrating a high concentration of component $\mathbf{5}$ at the area exposed during the first illumination step. Calibration experiments, ${ }^{35}$ which are presented in the Supporting Information, Figure S5, revealed the relation between the intensity ratio $I_{1410} /\left(I_{1410}+I_{2226}\right)$ and the actual concentration of azobenzene and liquid-crystal 3.

For polymerization-induced diffusion experiments, we have chosen to compare the reactive azobenzene $\mathbf{4}$ and $\mathbf{5}$ to address some molecular structural elements in the polymerizationinduced diffusion process. Azobenzene diacrylate 4 has a relatively long aliphatic spacer, to support smectic phase formation, and a polymerizable acrylate group with intrinsically similar polymerization properties to those of the other acrylates of the mixture. Azobenzene dimethacrylate 5 differs in two respects. It has a much shorter spacer length, making the total size of the molecule smaller, and therefore will demonstrate a larger diffusivity in the reaction mixture. The methacrylate function is known to have a higher reactivity ratio in an acrylate environment. This property will enhance the higher chemical potential for the monomer to diffuse to the high-intensity areas during mask exposure. However, unlike traditional studies on polymerization-induced diffusion processes, here the polymerization takes place in the smectic phase, which might affect diffusion and polymerization kinetics.

In the first experiment, we deduced that the local azobenzene 4 concentration in the illuminated spot region is increased from 7.4 to $9.6 \mathrm{wt} \%$ during masked exposure. In the masked region, where polymerization is prevented, the azobenzene concentration decreases to $6.4 \mathrm{wt} \%$.

In the next experiment, we replace the azobenzene diacrylate 4 with the azobenzene dimethacrylate 5 . Now the azobenzene concentration in the illuminated area increases from 5.2 to 7.2 wt $\%$, which makes the diffusion results between the two azobenzene monomers largely comparable. The local enrichment of azobenzene may depend on various other factors such as temperature and especially also on the intensity of the first exposure step, which determines the balance between diffusion time and fixation in the polymerization process. ${ }^{28}$ The polymerization temperature is largely determined by the temperature range of the smectic phase that we would like to polymerize in. The light intensity of the maskwise exposure was varied between 0 and $1.2 \mathrm{~mW} / \mathrm{cm}^{2}$. Between 0.1 and 1.2 $\mathrm{mW} / \mathrm{cm}^{2}$, the difference in azobenzene is only marginal with somewhat higher azobenzene concentrations found for the low intensities as shown in the Supporting Information, Figure S6. Below $0.1 \mathrm{~mW} / \mathrm{cm}^{2}$, the polymerization drops to very low values because of inhibition effects caused by added stabilizers and dissolved oxygen. At higher intensities above $1.2 \mathrm{~mW} / \mathrm{cm}^{2}$, the polymerization is completed before full diffusion could be completed.

To estimate whether the enhanced azobenzene concentrations alone could be responsible for the contrast in liquid secretion, a control experiment is performed where the area that is polymerized during the second exposure is in its smectic state rather than heating it to the isotropic state. Figure $4 a, b$ 
shows the resulting DHM and POM images. The protrusions in the illuminated area during maskwise exposure correlate with the higher azobenzene concentration area (further referred to as azo-rich) of the Raman measurement, confirming material diffusion. Also, the color contrast of the optical microscope points to a higher azobenzene concentration in the same area. AFM measurements showing a somewhat small pore size in the azo-rich areas and higher modulus further support our model on material diffusion, especially on the diacrylates (Supporting information, Figure S7).

When these samples are exposed to UV light to induce secretion, first, the liquid is ejected from the azo-rich area as is the case with the earlier samples. However, under continued exposure, this contrast is lost to some extent, which means the local secretion can only be limited to small volumes as is seen in Figure 4a,b (Movies S5 and S6), which shows the maximum volume that is secreted under the condition of local exclusivity. Note that these secretion values are a factor of 10 lower than that in the isotropic case next to the smectic area (Figures $2 \mathrm{a}$ and $4 \mathrm{c}$ ).

\section{CONCLUSIONS}

We have demonstrated that we are capable of controlling fluid release from a liquid-embedded porous $\mathrm{LCN}$ coating at predesignated locations. Liquid is released through a controlled contraction of the pores by a squeezing mechanism of the LCN. To localize this secretion at the desired location, we create a coating with hybrid smectic and nonordered isotropic molecular alignments. This coating is fabricated by a maskwise exposure to fix the smectic order followed by a flood exposure by heating up the coating in the isotropic phase. Upon activation, liquid is exclusively rejected from the area with the smectic order. We further discovered that the light-responsive molecule, azobenzene, predominantly exists in the smectic region caused by the polymerization-induced diffusion process during the first maskwise step. We anticipate that the precise liquid rejection at the desired location at the coating surface can be used in a variety of applications ranging from liquefying surfaces for cleaning, de-icing, and drug release for medical applications, to friction control in robotic devices.

\section{MATERIALS AND METHODS}

Materials. Monomers 1, 2, and 5 were kindly supplied by Philips Research Organics and used as received. The nonreactive mesogen 3 and monomer 4 were purchased from Synthon and photoinitiator 6 (Irgacure 819) from Ciba. The coating consisting of molecule 4 was prepared with a mixture that contained $13.8 \mathrm{wt} \%$ diacrylate $1,13.8 \mathrm{wt}$ $\%$ monoacrylate 2, 64.0 wt \% molecule 3, 7.4 wt \% azobenzene diacrylate 4 , and $1.0 \mathrm{wt} \%$ photoinitiator 6 . The coating consisting of molecule 5 was prepared with a mixture that contained 14.9 wt \% diacrylate 1, 14.9 wt \% monoacrylate 2, 64.0 wt \% nonreactive molecule 3, 5.2 wt \% azobenzene methacrylate 5, and 1.0 wt \% photoinitiator 6 . The liquid-crystal monomer mixtures were prepared by dissolving in dichloromethane. Chemical structures of the materials are shown in Scheme 1.

Sample Preparation. Glass substrates were cleaned using acetone and isopropanol for $20 \mathrm{~min}$ in an ultrasonic water bath, respectively. Then, UV-ozone treatment was used to clean the substrate surface for $20 \mathrm{~min}$. One glass substrate was dip-coated with a monolayer of 3(trimethoxysilyl)propyl methacrylate to enhance the adhesion, and the other glass plate was coated with a thin polyimide (SE5661, Nissan) layer. A cell was formed by gluing these two glass plates. A coating with a uniform smectic molecular alignment was fabricated by heating the mixture to the isotropic phase and then filling into the cells by capillary forces. The sample was cooled to the smectic phase
Scheme 1. Composite Used to Fabricate the Coating ${ }^{a}$<smiles>C=CC(=O)OC1(C)CCCC1Oc1ccc(C(=O)Oc2ccc(OC(=O)C3CCC(Oc4ccccc4)CC3)cc2)cc1</smiles>

2<smiles>C=CC(=O)OC1(C)CCCC1OC(=O)C1CCC(OC(=O)c2ccc(OC(=O)c3ccc(OCCCCCC)cc3)cc2)CC1</smiles>

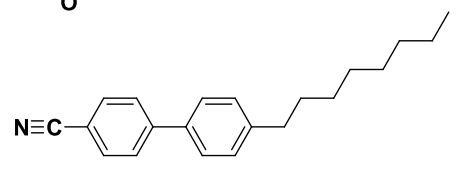

4

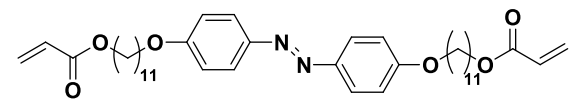

5

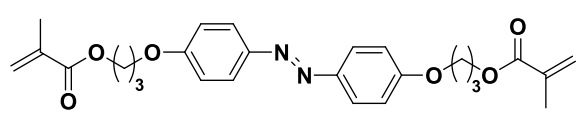

6

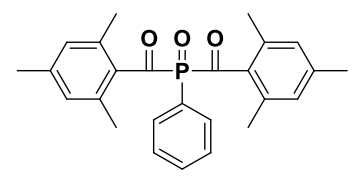

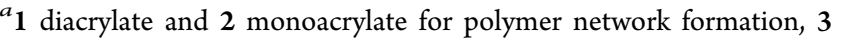
nonreactive liquid-crystal molecule acting as the porogen, 4 azobenzene diacrylate, 5 azobenzene methacrylate inducing photomechanical responsiveness, and 6 photoinitiator that can be excited at the wavelength of $>400 \mathrm{~nm}$.

at $35{ }^{\circ} \mathrm{C}$ and cured by ultraviolet exposure using a mercury lamp (EXFO Omnicure S2000). A cutoff filter with transmitting light $>400$ $\mathrm{nm}$ (Newport FSQ-GG400 filter) was used during the polymerization to prevent premature isomerization of azobenzene. After polymerization, the cell was opened with a razor blade, and the final coating thickness was approximately $20 \mu \mathrm{m}$.

Sample Characterization. Molecular alignment and excretion behavior of the coating were investigated by a polarized optical microscope (Nikon Ci Eclipse). The thickness of the coating was measured by interferometry (Fogale NanoTech zoomsurf). Liquid secretion at the coating surface was measured by digital holography microscopy (Lyncée Tec DHM-R2100) and data were processed using Koala software. The cross section of the LCN coating was prepared after extraction with cyclohexane by cryogenic cutting in liquid nitrogen and measured with a scanning electron microscope (FEI SEM Quanta 3D FEG) in secondary electron mode. The nanoscale elastic modulus and height were measured by a Multimode atomic force microscope in PeakForce Quantitative Nanomechanical Mapping (PF-QNM) mode (Bruker Corporation) using HQ:NSC19/ No Al (MikroMasch) cantilevers. Confocal Raman measurements were performed at room temperature using a Witec $\alpha$-300 R $\mu$-Raman system. For $2 \mathrm{D}$ surface mapping, a $200 \mu \mathrm{m} \times 200 \mu \mathrm{m}$ sampling area in patterned coatings was mapped with an array of $50 \times 50$ spectra (2500 data points or pixels) meaning $4 \mu \mathrm{m} \times 4 \mu \mathrm{m}$ pixel size in $X$ and $Y$ directions, while a $50 \mu \mathrm{m} \times 50 \mu \mathrm{m}$ sample area with an array of 50 $\times 50$ spectra was investigated for reference samples without any protrusions. In Raman maps, 50x objective lens with a numerical aperture of 0.55 was utilized together with $2 \mathrm{~s}$ integration time, 600 $\mathrm{mm} / \mathrm{g}$ grating, $785 \mathrm{~nm}$ continuous laser, and $40 \mathrm{~mW}$ laser power. The penetration depth of the laser was $3.8 \mu \mathrm{m}$. Prior to Raman data analysis, spike (or cosmic ray) removal and background subtraction were performed using Project 5 software. 


\section{ASSOCIATED CONTENT}

\section{(s) Supporting Information}

The Supporting Information is available free of charge at https://pubs.acs.org/doi/10.1021/acsapm.0c00691.

Interferometry measurements of coating thickness prior to and after solvent extraction; POM image of the isotropic coating; Raman spectra of coatings; Raman calibration curves of azobenzene and liquid; Raman measurement of material diffusion; and AFM measurement of coating surfaces (PDF)

Polarized optical microscopy movie of light-triggered uniform liquid secretion (MP4)

Digital holographic microscopy movie of light-triggered uniform liquid secretion (MP4)

Optical microscopy movie of light-triggered localized liquid secretion at the preset position of the coating having a hybrid molecular configuration (MP4)

Digital holographic microscopy movie of light-triggered localized liquid secretion at the preset position of the coating having a hybrid molecular configuration (MP4)

Digital holographic microscopy movie of light-triggered liquid secretion of the coating with a smectic alignment (MP4)

Optical microscopy movie of light-triggered liquid secretion of the coating with a smectic alignment (MP4)

\section{AUTHOR INFORMATION}

\section{Corresponding Authors}

Guofu Zhou - SCNU-TUE Joint Lab of Device Integrated Responsive Materials (DIRM), National Center for International Research on Green Optoelectronics, South China Normal University, Guangzhou 510006, China; 이이.org/ 0000-0003-1101-1947; Email: guofu.zhou@m.scnu.edu.cn

Danqing Liu - SCNU-TUE Joint Lab of Device Integrated Responsive Materials (DIRM), National Center for International Research on Green Optoelectronics, South China Normal University, Guangzhou 510006, China; Laboratory for Functional Organic Materials and Devices (SFD), Department of Chemical Engineering and Chemistry and Institute for Complex Molecular Systems (ICMS), Eindhoven University of Technology, 5612 AE Eindhoven, The Netherlands; ○ orcid.org/0000-0001-8830-0443; Email: D.Liu1@tue.nl

\section{Authors}

Yuanyuan Zhan - SCNU-TUE Joint Lab of Device Integrated Responsive Materials (DIRM), National Center for International Research on Green Optoelectronics, South China Normal University, Guangzhou 510006, China; Laboratory for Functional Organic Materials and Devices (SFD), Department of Chemical Engineering and Chemistry and Institute for Complex Molecular Systems (ICMS), Eindhoven University of Technology, 5612 AE Eindhoven, The Netherlands

Wanshu Zhang - Laboratory for Functional Organic Materials and Devices (SFD), Department of Chemical Engineering and Chemistry, Eindhoven University of Technology, 5612 AE Eindhoven, The Netherlands

Ayberk Özden - Laboratory for Functional Organic Materials and Devices (SFD), Department of Chemical Engineering and Chemistry, Eindhoven University of Technology, 5612 AE Eindhoven, The Netherlands
Simon Houben - Laboratory for Functional Organic Materials and Devices (SFD), Department of Chemical Engineering and Chemistry, Eindhoven University of Technology, 5612 AE Eindhoven, The Netherlands; (1) orcid.org/0000-0002-9158056X

Margarita Schuster - Research Group Zentel Organic/ Macromolecular Chemistry, Institute for Organic Chemistry, Johannes Gutenberg-University Mainz, 55122 Mainz, Germany

Hubert Gojzewski - Materials Science and Technology of Polymers, Faculty of Science and Technology, University of Twente, 7522 NB Enschede, The Netherlands; (1) orcid.org/ 0000-0001-6325-8293

Dirk J. Broer - SCNU-TUE Joint Lab of Device Integrated Responsive Materials (DIRM), National Center for International Research on Green Optoelectronics, South China Normal University, Guangzhou 510006, China; Laboratory for Functional Organic Materials and Devices (SFD), Department of Chemical Engineering and Chemistry and Institute for Complex Molecular Systems (ICMS), Eindhoven University of Technology, 5612 AE Eindhoven, The Netherlands

Complete contact information is available at:

https://pubs.acs.org/10.1021/acsapm.0c00691

\section{Author Contributions}

The manuscript was written through contributions of all authors. All authors have given approval to the final version of the manuscript.

\section{Notes}

The authors declare no competing financial interest.

\section{ACKNOWLEDGMENTS}

We thank for the financial support from the National Key R\&D Program of China (No. 2020YFE0100200), the Netherlands Organization for Scientific Research (NWO VENI grant 15135), the framework of the 4TU. High-Tech Materials research program "New Horizons in designer materials" (www.4tu.nl/htm), the Netherlands, Science and Technology Program of Guangzhou (No. 2019050001), Guangdong Provincial Key Laboratory of Optical Information Materials and Technology (No. 2017B030301007), MOE International Laboratory for Optical Information Technologies and the 111 Project.

\section{ABBREVIATIONS}

LCN, liquid-crystal polymer network; UV, ultra-violet; PDMS, poly(dimethylsiloxane); POM, polarized optical microscopy; SEM, scanning electron microscopy; DHM, digital holographic microscopy; AFM, atomic force microscopy

\section{REFERENCES}

(1) Cascales, E.; Christie, P. J. The Versatile Bacterial Type IV Secretion Systems. Nat. Rev. Microbiol. 2003, 1, 137-149.

(2) Chirico, W. J. Protein Release Through Nonlethal Oncotic Pores as an Alternative Nonclassical Secretory Pathway. BMC Cell Biol. 2011, 12, 46.

(3) Hancock, R. E. W.; Scott, M. G. The Role of Antimicrobial Peptides in Animal Defenses. Proc. Natl. Acad. Sci. U.S.A. 2000, 97, $8856-8861$.

(4) Tattersall, I. Human-Biology - an Introduction to HumanEvolution, Variation, Growth, and Adaptability, 3rd Edition Harrison,Ga, Tanner,Jm, Pilbeam,Dr, Baker,Pt. Nature 1989, 338, 184. 
(5) Goatley, J. L.; Lewis, R. W. Composition of Guttation Fluid from Rye Wheat and Barley Seedlings. Plant Physiol. 1966, 41, 373-375.

(6) Ellis, A. E. Innate Host Defense Mechanisms of Fish Against Viruses and Bacteria. Dev. Comp. Immunol. 2001, 25, 827-839.

(7) Koziel, J.; Potempa, J. Protease-Armed Bacteria in the Skin. Cell Tissue Res. 2013, 351, 325-337.

(8) Cui, J.; Daniel, D.; Grinthal, A.; Lin, K.; Aizenberg, J. Dynamic Polymer Systems with Self-Regulated Secretion for the Control of Surface Properties and Material Healing. Nat. Mater. 2015, 14, 790795.

(9) Wong, T. S.; Kang, S. H.; Tang, S. K. Y.; Smythe, E. J.; Hatton, B. D.; Grinthal, A.; Aizenberg, J. Bioinspired Self-Repairing Slippery Surfaces with Pressure-Stable Omniphobicity. Nature 2011, 477, 443-447.

(10) Howell, C.; Grinthal, A.; Sunny, S.; Aizenberg, M.; Aizenberg, J. Designing Liquid-Infused Surfaces for Medical Applications: A Review. Adv. Mater. 2018, 30, No. 1802724.

(11) Gelebart, A. H.; Liu, D.; Mulder, D. J.; Leunissen, K. H. J.; van Gerven, J.; Schenning, A. P. H. J.; Broer, D. J. Photoresponsive Sponge-Like Coating for On-Demand Liquid Release. Adv. Funct. Mater. 2018, 28, No. 1705942.

(12) Pang, X.; Lv, J.; Zhu, C.; Qin, L.; Yu, Y. Photodeformable Azobenzene-Containing Liquid Crystal Polymers and Soft Actuators. Adv. Mater. 2019, 31, No. 1904224.

(13) Gelebart, A. H.; Mc Bride, M.; Schenning, A. P. H. J.; Bowman, C. N.; Broer, D. J. Photoresponsive Fiber Array: Toward Mimicking the Collective Motion of Cilia for Transport Applications. Adv. Funct. Mater. 2016, 26, 5322-5327.

(14) Yu, Y.; Nakano, M.; Ikeda, T. Directed Bending of a Polymer Film by Light. Nature 2003, 425, 145.

(15) Iamsaard, S.; Asshoff, S. J.; Matt, B.; Kudernac, T.; Cornelissen, J. J. L. M.; Fletcher, S. P.; Katsonis, N. Conversion of Light into Macroscopic Helical Motion. Nat. Chem. 2014, 6, 229-235.

(16) Palffy-Muhoray, P. Liquid Crystal Elastomers and Light. Adv. Polym. Sci. 2012, 250, 95-118.

(17) Zeng, H.; Wani, O. M.; Wasylczyk, P.; Kaczmarek, R.; Priimagi, A. Self-Regulating Iris Based on Light-Actuated Liquid Crystal Elastomer. Adv. Mater. 2017, 29, No. 1701814.

(18) Wani, O. M.; Zeng, H.; Priimagi, A. A light-Driven Artificial Flytrap. Nat. Commun. 2017, 8, No. 15546.

(19) Ma, S.; Li, X.; Huang, S.; Hu, J.; Yu, H. A Light-Activated Polymer Composite Enables On-Demand Photocontrolled Motion: Transportation at the Liquid/Air Interface. Angew. Chem., Int. Ed. 2019, 58, 2655-2659.

(20) Liu, D.; Broer, D. J. Self-assembled Dynamic 3D Fingerprints in Liquid-Crystal Coatings Towards Controllable Friction and Adhesion. Angew. Chem., Int. Ed. 2014, 53, 4542-4546.

(21) Feng, W.; Broer, D. J.; Liu, D. Combined Light and Electric Response of Topographic Liquid Crystal Network Surfaces. Adv. Funct. Mater. 2020, 30, No. 1704970.

(22) Liu, D.; Broer, D. J. New Insights into Photoactivated Volume Generation Boost Surface Morphing in Liquid Crystal Coatings. Nat. Commun. 2015, 6, No. 8334

(23) Yamada, M.; Kondo, M.; Mamiya, J. I.; Yu, Y. L.; Kinoshita, M.; Barrett, C. J.; Ikeda, T. Photomobile Polymer Materials: Towards Light-Driven Plastic Motors. Angew. Chem., Int. Ed. 2008, 47, 49864988.

(24) White, T. J.; Tabiryan, N. V.; Serak, S. V.; Hrozhyk, U. A.; Tondiglia, V. P.; Koerner, H.; Vaia, R. A.; Bunning, T. J. A High Frequency Photodriven Polymer Oscillator. Soft Matter 2008, 4, 1796-1798.

(25) Lan, R.; Sun, J.; Shen, C.; Huang, R.; Zhang, Z.; Ma, C.; Bao, J.; Zhang, L.; Wang, L.; Yang, D.; Yang, H. Light-Driven Liquid Crystalline Networks and Soft Actuators with Degree-of-FreedomControlled Molecular Motors. Adv. Funct. Mater. 2020, No. 2000252.

(26) Liu, D. Volume Generation towards Dynamic Surface Morphing in Liquid Crystal Polymer Networks. Liq. Cryst. 2016, 43, 2136-2143.
(27) Leewis, C. M.; de Jong, A. M.; van IJzendoorn, L. J.; Broer, D. J. Reaction-Diffusion Model for the Preparation of Polymer Gratings by Patterned Ultraviolet Illumination. J. Appl. Phys. 2004, 95, 41254139.

(28) Sánchez, C.; de Gans, B. J.; Kozodaev, D.; Alexeev, A.; Escuti, M. J.; van Heesch, C.; Bel, T.; Schubert, U. S.; Bastiaansen, C. W. M.; Broer, D. J. Photoembossing of Periodic Relief Structures Using Polymerization-Induced Diffusion: A Combinatorial Study. Adv. Mater. 2005, 17, 2567-2571.

(29) Hermans, K.; Harnidi, S. Z.; Spoelstra, A. B.; Bastiaansen, C. W. M.; Broer, D. J. Rapid, Direct Fabrication of Antireflection-Coated Microlens Arrays by Photoembossing. Appl. Opt. 2008, 47, 65126517.

(30) Hisano, K.; Kurata, Y.; Aizawa, M.; Ishizu, M.; Sasaki, T.; Shishido, A. Alignment Layer-Free Molecular Ordering Induced by Masked Photopolymerization with Non-Polarized Light. Appl. Phys. Express 2016, 9, No. 072601.

(31) Hisano, K.; Aizawa, M.; Ishizu, M.; Kurata, Y.; Nakano, W.; Akamatsu, N.; Barrett, C. J.; Shishido, A. Scanning Wave Photopolymerization Enables Dye-Free Alignment Patterning of Liquid Crystals. Sci. Adv. 2017, 3, No. e1701610.

(32) Liu, D.; Bastiaansen, C. W. M.; den Toonder, J. M. J.; Broer, D. J. Single-Composition Three-Dimensionally Morphing Hydrogels. Soft Matter 2013, 9, 588-596.

(33) Liu, D.; Bastiaansen, C. W. M.; den Toonder, J. M. J.; Broer, D. J. Photo-)Thermally Induced Formation of Dynamic Surface Topographies in Polymer Hydrogel Networks. Langmuir 2013, 29, $5622-5629$.

(34) Broer, D. J.; Lub, J.; Mol, G. N. Wide-Band Reflective Polarizers from Cholesteric Polymer Networks with a Pitch Gradient. Nature 1995, 378, 467-469.

(35) Samek, O.; Obruca, S.; Siler, M.; Sedlacek, P.; Benesova, P.; Kucera, D.; Marova, I.; Jezek, J.; Bernatova, S.; Zemanek, P. Quantitative Raman Spectroscopy Analysis of Polyhydroxyalkanoates Produced by Cupriavidus Necator H16. Sensors 2016, 16, 1808. 